\title{
Atuação do cirurgião-dentista no atendimento à adolescentes no Brasil
}

\author{
Performance of the dentist in assisting adolescents in Brazil \\ Desempeño del dentista en la asistencia a los adolescentes em Brasil
}

Recebido: 25/10/2021 | Revisado: 04/11/2021 | Aceito: 14/11/2021 | Publicado: 24/11/2021

\author{
Dayane Esteves \\ ORCID: https://orcid.org/0000-0002-8673-4026 \\ Universidade Federal de Alfenas, Brasil \\ E-mail: dayesteves26@gmail.com \\ François Isnaldo Dias Caldeira \\ ORCID: https://orcid.org/0000-0002-4688-2059 \\ Universidade Estadual Paulista, Brasil \\ E-mail: fraqncoisdias@hotmail.com \\ Ihanael Ifanger \\ ORCID: https://orcid.org/0000-0002-4046-4913 \\ Universidade Federal de Alfenas, Brasil \\ E-mail: ihanaifanger@gmail.com \\ Daniela Coelho de Lima \\ ORCID: https://orcid.org/0000-0002-7945-435X \\ Universidade Federal de Alfenas, Brasil \\ E-mail: danielaclunifal@gmail.com \\ Clícia Valim Côrtes Gradim \\ ORCID: https://orcid.org/0000-0002-1852-2646 \\ Universidade Federal da Paraíba. Brasil \\ E-mail: clicia.gradim@academico.ufpb.br
}

\begin{abstract}
Resumo
Identificar a produção científica acerca de pesquisas sobre ao atendimento de adolescentes na Odontologia brasileira. Foram realizadas buscas virtuais nos bancos de dados PubMed, BIREME, Web of Sciences, LILACS e BBO, no período de setembro de 2020. Foi observado que 34.8\% dos artigos publicados se concentravam nas regiões Nordeste e Sudeste. Em relação aos eixos temáticos abordados pelas presentes investigações pode-se perceber que, $43.5 \%$ dos artigos destacavam a necessidade de acesso aos serviços de saúde bucal. $17.4 \%$ evidenciaram a prevalência e necessidades de cuidados com a saúde bucal e $17.4 \%$ associaram o diagnóstico e a incidência das comorbidades. Diante disso, é necessário que os centros de pesquisas multicêntricas e a estratégia de saúde da família adotem medidas preventivas e de promoção em saúde considerando os indicadores socioeconômicos de cada região promovendo assim, o elo entre o cirurgião-dentista e o paciente adolescente.
\end{abstract}

Palavras-chave: Adolescente; Dentista; Saúde pública.

\begin{abstract}
To identify the scientific production about research on adolescent care in Brazilian dentistry. Virtual searches were conducted in the PubMed, BIREME, Web of Sciences, LILACS and BBO databases from September 2020. It was observed that $34.8 \%$ of the published articles were concentrated in the Northeast and Southeast regions. In relation to the thematic axes addressed by the present investigations it can be noticed that, $43.5 \%$ of the articles highlighted the need for access to oral health services. $17.4 \%$ highlighted the prevalence and needs of oral health care and $17.4 \%$ associated the diagnosis and incidence of comorbidities. Therefore, it is necessary that multicenter research centers and the family health strategy adopt preventive and health promotion measures considering the socioeconomic indicators of each region, thus promoting the link between the dental surgeon and the adolescent patient.
\end{abstract}

Keywords: Adolescent; Dentist; Public health.

\section{Resumen}

Identificar la producción científica en relación con las investigaciones sobre la atención a los adolescentes en la odontología brasileña. Se realizaron búsquedas virtuales en las bases de datos PubMed, BIREME, Web of Sciences, LILACS y BBO durante el periodo de septiembre de 2020. Se observó que el 34,8\% de los artículos publicados se concentraban en las regiones del noreste y el sureste. En cuanto a los ejes temáticos abordados por las presentes investigaciones se puede observar que, el 43,5\% de los artículos destacaron la necesidad de acceso a los servicios de salud bucodental. El 17,4\% mostró la prevalencia y las necesidades de atención a la salud bucodental y el 17,4\% asoció el diagnóstico y la incidencia de comorbilidades. Por lo tanto, es necesario que los centros de investigación multicéntricos y la estrategia de salud familiar adopten medidas de prevención y promoción de la salud considerando los indicadores socioeconómicos de cada región, promoviendo así el vínculo entre el cirujano dentista y el paciente adolescente.

Palabras clave: Adolescente; Dentista; Salud pública. 


\section{Introdução}

A prevenção no Brasil é uma temática relativamente nova, pois o seu marco ocorreu com a implantação dos programas de prevenção pelo Sistema único de Saúde (SUS). Essa modalidade de assistência que visa a prevenção e a promoção da saúde tem na equipe multidisciplinar o seu eixo e como base os Programas de Saúde da Família (PSF), local onde o cirurgião dentista foi alocado na equipe no final dos anos 2000 como membro da equipe de atenção primária.

O cirurgião dentista entra na equipe de atenção primária com a proposta de tentar melhorar a prevenção de cáries e da melhora da saúde bucal na Estratégia de Saúde da Família (ESF). Importante salientar que o cirurgião dentista atuava nas escolas desde a década de 60 com um perfil curativista e de atendimento de pacientes com queixas. Essa inserção na atenção primária acarretou em uma reformulação de suas práticas, em consequência, da mudança da visão biomédica para a biopsicossocial, fazendo com que o cirurgião-dentista repensasse suas atividades, valores e conhecimentos envolvidos na saúde (Ely et al., 2016; Emerich et al., 2015; Taquette, 2010).

$\mathrm{Na}$ Odontologia, mesmo com o desenvolvimento do aparato tecnológico que ocorreu no século XX, pôde-se perceber que esse modelo curativista esteve apenas eliminando a doença instalada, não controlava e nem evitava sua reincidência (Assaf et al., 2014; Souza \& Roncalli, 2007).

A adolescência que corresponde ao período de mudanças físicas e psicológicas que corresponde a faixa etária entre 14 a 19 anos, observa-se que o adolescente tende a se preocupar com o autocuidado e com sua imagem corporal para aceitação do grupo e de relacionamento. Frente a isso passa a ter um cuidado maior com a boca, pois, a fala e o início da vida amorosa tem influência nesse cuidado (Spezzia et al, 2017).

Em nossa realidade de prática de campo no curso de Odontologia da Universidade, a mesma oferece ao acadêmico o atendimento junto a equipe dos Programas de Saúde da Família do município. Durante o desenvolvimento dessas atividades verificou-se que os adolescentes procuravam o serviço para o atendimento com ênfase em promoção e estética. Esse fator nos levou ao interesse se esse fator também ocorria em outras regiões do país, o que incentivou a realização desse estudo.

\section{Metodologia}

A presente revisão integrativa, seguiu o rigor metodológico descrito por Mendes, Silveira e Galvão, que definem essa metodologia como uma análise que inclui pesquisas significantes que dão suporte para a resolução e melhoramento da prática clínica, além de identificar falhas do conhecimento que precisam ser preenchidas com a realização de novas pesquisas. Para isso, foram realizadas as seguintes etapas:1) Identificação do tema e seleção da hipótese, 2) Estabelecimento de critérios para inclusão e exclusão de estudos, 3) Definição das informações a serem extraídas dos estudos selecionados/ categorização dos estudos, 4) Avaliação dos estudos incluídos na revisão integrativa, 5) Interpretação dos resultados e 6) Apresentação da revisão/síntese do conhecimento (Mendes et al., 2008).

Para alcançar o objetivo proposto utilizou-se a pergunta norteadora: qual o papel do cirurgião dentista no atendimento aos adolescentes nos de saúde e ensino públicos no Brasil. Para a construção da pergunta norteadora o acrônimo PICO, em que o P representa o paciente, o I a intervenção, o C a comparação e o O o desfecho, esses são componentes que auxiliam a busca bibliográfica e as evidências encontradas. Assim, nesse estudo o P se refere aos adolescentes, o I as intervenções do cirurgião dentista, o $\mathrm{C}$ ao contexto de atendimento do cirurgião dentista e o $\mathrm{O}$ ao atendimento nos serviços de saúde ou de ensino (Santos et al, 2006).

Foram realizados os bancos da Pub med, Web of Science, BIREME, LILACS e BBO para publicações no período de 2010 até setembro de 2020. Os artigos relevantes foram identificados através de pesquisas pelos descritores em língua inglesa do Medical Subject Headings (MeSH) utilizando o operador Booleano "and" entre os descritores "dentista and adolescente and Saúde Pública" e "Dentist and Adolescent and Public Health". 
As buscas bibliográficas realizadas nos bancos de dados eletrônicos foram exportadas para o programa EndNote Program $^{\mathrm{TM}}$ versão X7 (Thomson Reuters, Nova Iorque, NY, EUA), a fim de eliminar referências duplicadas. Dois investigadores (D.M e F.I.C.D.) realizaram a pesquisa inicial para avaliação dos títulos e resumos de forma independente, e os resultados foram verificados para o acordo com base nos critérios de seleção. Para avaliações conflitantes, chegou-se a um acordo após uma discussão que incluiu um terceiro pesquisador (C.V.C.G.).

Foram aplicados os seguintes critérios de inclusão: Artigos nos idiomas português e inglês; pesquisas realizadas no Brasil; temáticas que abordassem o atendimento do paciente adolescente; relato de dados sobre a idade do adolescente (10 a 20 anos). Foram excluídos artigos em espanhol; cartas; relatos de experiência; estudos de casos, dissertações, teses, livros e artigos que não foram disponibilizados na integra.

Após a organização das informações a serem extraídas dos estudos selecionados, ou seja, os estudos foram catalogados de acordo com o instrumento de URSI, 2005 e foram analisados utilizando o nível de evidencia descritos Melnyk e FineoutOverholt, 2011. São eles: Nível 1 - metanálise de múltiplos estudos controlados, Nível 2 - estudo individual com delineamento experimental. Nível 3 - estudo com delineamento quase experimental, nível 4 - estudos experimentais estudo com delineamento não-experimental como pesquisa descritiva correlacional e qualitativa ou estudos de caso; nível 5, relatório de casos ou dado obtido de forma sistemática, de qualidade verificável ou dados de avaliação de programas; nível 6 - estudos qualitativos e nível 7, opinião de autoridades respeitáveis baseada na competência clínica ou opinião de comitês de especialistas, incluindo interpretações de informações não baseadas em pesquisas. (Melnyk \& Fineout-Overholt, 2011; Ursi, 2005).

Inicialmente, foram encontrados 2045 estudos potenciais para esta revisão nas diversas bases. No entanto, ao realizar a exclusão das duplicatas houve uma redução para 1611 artigos. Esses foram analisados por título e resumo e 1572 foram excluídos por não responder o objetivo da pesquisa; foram elegíveis 39 artigos para análise na íntegra. Após a leitura, 16 artigos foram excluídos por não responderem à pergunta norteadora; sendo eleitos 22 artigos para o trabalho final. A Figura 1 demonstra a síntese do processo de seleção dos artigos utilizando o fluxograma do PRISMA.

Figura 1 - Fluxograma da busca de informações do estudo.
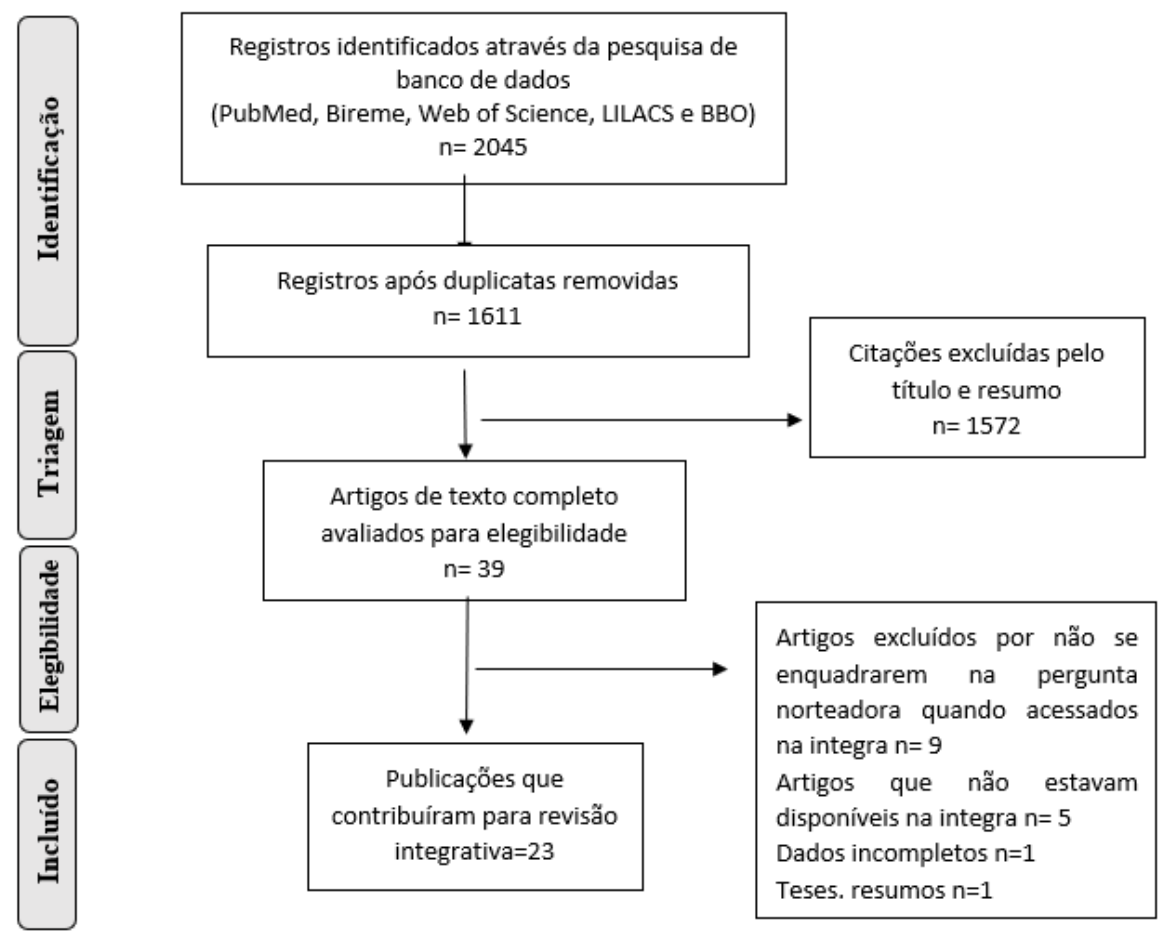

Fonte: Autores. 


\section{Resultados}

O Quadro 1 apresenta os estudos incluídos na revisão integrativa acerca da experiência do Cirurgião-Dentista no atendimento dos adolescentes brasileiros.

Verifica-se que em relação ao tipo de estudo $95.62 \%(n=22)$ deles estão enquadrados no nível de evidência cinco, o que indica que foram estudo descritos e com amostra bem delineada. Em relação ao período de publicação dos estudos verificouse que em todos os anos tem pelo menos um artigo, sendo os anos de 2014 e 2015 com maior número de publicações, quatro $(17 \%)$ e sete $(30 \%)$ respectivamente, sendo os dois anos com maior publicação como pode ser verificado no Quadro 1.

Quadro 1 - Dados sobre os estudos no que se refere a autoria, Título, ano, estado, amostra por sexo, objetivo, conclusão e nível de evidência.

\begin{tabular}{|c|c|c|c|c|c|}
\hline & $\begin{array}{l}\text { Autor, nome do artigo, ano e } \\
\text { estado da federação }\end{array}$ & $\begin{array}{c}\text { Faixa } \\
\text { etária e } \\
\text { Amostra por } \\
\text { sexo } \\
\end{array}$ & Objetivo & Resultados & N.E \\
\hline 2 & $\begin{array}{l}\text { Assaf et al., Dental caries in inland } \\
\text { Brazilian adolescents and its } \\
\text { relationship with social } \\
\text { determinants. 2014. Rio de Janeiro }\end{array}$ & $\begin{array}{c}12 \\
\text { Mas: } 228 \\
\text { Fem: } 276\end{array}$ & $\begin{array}{c}\text { Identificar a prevalência de } \\
\text { cárie dentária em } \\
\text { adolescentes de } 12 \text { anos no } \\
\text { município de Nova Friburgo, } \\
\text { RJ, Brasil e analisar a } \\
\text { influência das variáveis } \\
\text { sociodemográficas, clínicas e } \\
\text { de acesso a serviços } \\
\text { odontológicos sobre a } \\
\text { experiência de cárie }\end{array}$ & $\begin{array}{l}\text { Observou-se baixa prevalência de cárie } \\
\text { dentária na faixa etária de } 12 \text { anos, com média } \\
\text { de } 1,90 \text { para o índice D3MFT, abaixo da média } \\
\text { nacional O percentual de indivíduos livres de } \\
\text { cárie ficou em torno de } 39,88 \% \text {, abaixo das } \\
\text { médias nacionais }(44 \%) \text { e estaduais }(50,6 \%) \text {. }\end{array}$ & 5 \\
\hline 3 & $\begin{array}{c}\text { Bendo et al., Social Vulnerability } \\
\text { and Traumatic Dental Injury } \\
\text { among Brazilian Schoolchildren: A } \\
\text { Population-Based Study. } 2012 . \\
\text { Minas Gerais } \\
\end{array}$ & $\begin{array}{c}11-14 \\
\text { Mas: } 649 \\
\text { Fem: } 907\end{array}$ & $\begin{array}{c}\text { Testar a associação entre } \\
\text { vulnerabilidade social e a } \\
\text { prevalência de lesão dentária } \\
\text { traumática (LDT) }\end{array}$ & $\begin{array}{c}\text { Identificou que o gênero masculino e } \\
\text { indivíduos pertencentes a áreas de maior } \\
\text { vulnerabilidade social são mais propensos a } \\
\text { apresentarem LDT. }\end{array}$ & 5 \\
\hline 4 & $\begin{array}{l}\text { Correa, Saintrain, Vieira, Saúde } \\
\text { bucal do adolescente: necessidades } \\
\text { odontológicas levantadas pelos } \\
\text { agentes comunitários de saúde. } \\
\text { 2011. Ceará. }\end{array}$ & $\begin{array}{c}10-19 \\
\text { Mas:310 } \\
\text { Fem: } 433\end{array}$ & $\begin{array}{c}\text { Apresentar dados } \\
\text { epidemiológicos relativos à } \\
\text { saúde bucal de adolescentes } \\
\text { obtidos com a utilização do } \\
\text { ICSB e levantados pelos } \\
\text { ACS em um município do } \\
\text { Estado do Ceará - Brasil }\end{array}$ & $\begin{array}{c}\text { Os indicadores de saúde bucal nos } \\
\text { adolescentes, foram baixos encontrando mais } \\
\text { de } 80 \% \text { com cárie visível; sendo que os } \\
\text { menores de } 12 \text { anos apresentaram condição } \\
\text { bucal mais grave que os adolescentes mais } \\
\text { velhos. } \\
\text { Dos entrevistados, } 51,3 \% \text { visitaram o dentista, } \\
\text { sendo que } 8,6 \% \text { por motivo emergencial. }\end{array}$ & 5 \\
\hline 5 & $\begin{array}{l}\text { Cunha et al., Association between } \\
\text { social conditions and oral health in } \\
\text { school failure .2019. São Paulo }\end{array}$ & $\begin{array}{c}15-19 \\
\text { Mas: } 2255 \\
\text { Fem: } 2907\end{array}$ & $\begin{array}{l}\text { Avaliar a associação do } \\
\text { fracasso escolar de } \\
\text { adolescentes brasileiros com } \\
\text { as condições sociais e } \\
\text { aspectos da saúde bucal por } \\
\text { meio de análise hierárquica. }\end{array}$ & $\begin{array}{c}\text { Distúrbios bucais e fatores sociais foram } \\
\text { associados ao fracasso escolar em } \\
\text { adolescentes. Uma trajetória escolar bem- } \\
\text { sucedida foi um forte determinante da saúde, } \\
\text { portanto, ações entre os setores de educação e } \\
\text { saúde devem ser desenvolvidas para os } \\
\text { adolescentes, principalmente aqueles com este } \\
\text { perfil. }\end{array}$ & 5 \\
\hline 6 & $\begin{array}{l}\text { da Fonseca et al., Factors } \\
\text { associated with last dental visit or } \\
\text { not to visit the dentist by Brazilian } \\
\text { adolescents: A population-based } \\
\text { study. 2017. São Paulo. }\end{array}$ & $\begin{array}{c}15-19 \\
\text { Mas: } 3039 \\
\text { Fem: } 2355\end{array}$ & $\begin{array}{c}\text { Investigar os fatores } \\
\text { associados à última visita } \\
\text { odontológica realizada há } \\
\text { três ou mais anos por } \\
\text { adolescentes no estado de } \\
\text { São Paulo, Brasil, utilizando } \\
\text { dados do Projeto de } \\
\text { Condições de Saúde Bucal } \\
\text { (SBSP-2015) }\end{array}$ & $\begin{array}{c}\text { (84,9\%) dos adolescentes relataram ter ido ao } \\
\text { dentista nos últimos } 2 \text { anos. Considerando que, } \\
626(11,6 \%) \text { não visitavam o dentista há mais } \\
\text { de } 3 \text { anos e } 188(3,4 \%) \text { nunca haviam visitado. } \\
\text { A condição bucal e dentária foi relatada como } \\
\text { satisfatória por } 4.350 \text { entrevistados }(80,6 \%) \text {, e } \\
\text { quando acessaram o serviço de saúde } 2.286 \\
(42,3 \%) \text { foram ao serviço público. }\end{array}$ & 5 \\
\hline 7 & $\begin{array}{c}\text { Dutra et al., Adolescents with } \\
\text { worse levels of oral health literacy } \\
\text { have more cavitated carious } \\
\text { lesions. 2019. Paraíba. }\end{array}$ & $\begin{array}{c}15-19 \\
\text { Mas: } 302 \\
\text { Fem: } 444\end{array}$ & $\begin{array}{l}\text { Investigar se a capacidade de } \\
\text { reconhecer e ler termos de } \\
\text { saúde bucal está associada ao } \\
\text { número de dentes com lesões } \\
\text { cariosas cavitadas em } \\
\quad \text { adolescentes. }\end{array}$ & $\begin{array}{c}\text { Adolescentes com níveis mais baixos de } \\
\text { alfabetização em saúde bucal tinham um maior } \\
\text { número de dentes com lesões cariadas } \\
\text { cavitadas do que aqueles com nível adequado } \\
\text { de alfabetização, independentemente de seu } \\
\text { status socioeconômico e história de visitar um } \\
\text { dentista }\end{array}$ & 5 \\
\hline 8 & Dutra et al., Degree of family & $15-19$ & Investigar associações entre & Os adolescentes com menor nível & 5 \\
\hline
\end{tabular}




\begin{tabular}{|c|c|c|c|c|c|}
\hline & $\begin{array}{l}\text { cohesion and social class are } \\
\text { associated with the number of } \\
\text { cavitated dental caries in } \\
\text { adolescents. 2020. Paraíba. }\end{array}$ & $\begin{array}{l}\text { Mas: } 302 \\
\text { Fem: } 444\end{array}$ & $\begin{array}{c}\text { cárie dentária e coesão } \\
\text { familiar, uso de drogas, } \\
\text { fatores sociodemográficos e } \\
\text { visitas ao dentista em } \\
\text { adolescentes de } 15 \text { a } 19 \text { anos }\end{array}$ & $\begin{array}{c}\text { socioeconômico e aqueles cuja coesão familiar } \\
\text { é classificada como desligada, separada ou } \\
\text { conectada apresentaram maior número de } \\
\text { lesões cavitadas. }\end{array}$ & \\
\hline 9 & $\begin{array}{l}\text { Ely et al., Impacto das equipes de } \\
\text { saúde bucal da Estratégia da Saúde } \\
\text { da Família na saúde bucal de } \\
\text { adolescentes do sul do Brasil. } \\
\text { 2015. Rio Grande do Sul. }\end{array}$ & $\begin{array}{l}12 \text { e } 15-19 \\
\text { Mas: } 3258 \\
\text { Fem: } 3804\end{array}$ & $\begin{array}{c}\text { Avaliar o impacto das } \\
\text { equipes de saúde bucal } \\
\text { (ESB) da Estratégia da Saúde } \\
\text { da Família (ESF) em } 2581 \\
\text { adolescentes escolares, com } \\
\text { idade de } 12 \text { e } 15-19 \text { anos de } \\
36 \text { municípios, } 19 \text { com e } 17 \\
\text { sem ESB/ESF }\end{array}$ & $\begin{array}{l}\text { No modelo bruto não houve associação das } \\
\text { ESB na ESF com nenhum dos desfechos } \\
\text { analisados. Após o ajuste em modelo } \\
\text { multinível, jovens de áreas não cobertas pela } \\
\text { ESB/ESF tiveram quase a metade da perda de } \\
\text { dentes dos adolescentes das áreas cobertas. }\end{array}$ & 5 \\
\hline 10 & $\begin{array}{l}\text { Emerich et al., Access to Dental } \\
\text { Services and Related Factors in } \\
\text { Adolescents from Vitória, Espírito } \\
\text { Santo, Brazil, 2011. 2015. Espírito } \\
\text { Santo. }\end{array}$ & $\begin{array}{c}15-19 \\
\text { Mas: } 172 \\
\text { Fem: } 259\end{array}$ & $\begin{array}{l}\text { Caracterizar o acesso dos } \\
\text { adolescentes aos serviços } \\
\text { odontológicos e identificar } \\
\text { seus determinantes }\end{array}$ & $\begin{array}{c}\text { A maioria dos entrevistados visita o dentista } \\
\text { pelo menos uma vez ao ano, tendo como } \\
\text { principal motivo a visita de rotina, ser atendida } \\
\text { em serviços públicos e avaliam positivamente } \\
\text { o serviço prestado. Os determinantes da } \\
\text { consulta são autopercepção de saúde bucal, } \\
\text { maior escolaridade e acesso a serviços } \\
\text { privados. }\end{array}$ & 5 \\
\hline 11 & $\begin{array}{c}\text { Fonseca, Wapniarz, Torres-Pereira, } \\
\text { Atitudes e acesso à informação de } \\
\text { saúde bucal de um grupo de } \\
\text { gestantes adolescentes. } 2014 . \\
\text { Paraná. }\end{array}$ & $\begin{array}{c}\text { 13-18 } \\
\text { Fem: } 38\end{array}$ & $\begin{array}{c}\text { Avaliar as atitudes das } \\
\text { gestantes adolescentes sobre } \\
\text { saúde bucal, bem como } \\
\text { alguns indicadores de acesso } \\
\text { e informação à atenção } \\
\text { odontológica nesta } \\
\text { população. } \\
\end{array}$ & $\begin{array}{c}\text { Apesar da maioria das adolescentes terem } \\
\text { visitado o Cirurgião-Dentista durante a } \\
\text { gestação, as consultas ocorreram na maior } \\
\text { parte por motivos emergenciais e não como } \\
\text { rotina. }\end{array}$ & 5 \\
\hline 12 & $\begin{array}{l}\text { Fontanini, Marshman, Vettore. } \\
\text { Social support and social network } \\
\text { as intermediary social determinants } \\
\text { of dental caries in adolescents. } \\
\text { 2015. Mato Grosso do Sul. }\end{array}$ & $\begin{array}{c}11-14 \\
\text { Mas:268 } \\
\text { Fem:268 }\end{array}$ & $\begin{array}{l}\text { Investigar a associação de } \\
\text { apoio social e rede social } \\
\text { com a cárie dentária de } \\
\text { adolescentes utilizando uma } \\
\text { versão adaptada da estrutura } \\
\text { conceitual dos determinantes } \\
\text { sociais de saúde da } \\
\text { Organização Mundial de } \\
\text { Saúde. } \\
\end{array}$ & $\begin{array}{l}\text { A saúde bucal dos adolescentes é influenciada } \\
\text { pelo número de redes sociais da família, bem } \\
\text { como pelos níveis de percepção social de apoio } \\
\text { proveniente de redes familiares. }\end{array}$ & 5 \\
\hline 13 & $\begin{array}{l}\text { Garcia et al., Conhecimento } \\
\text { Odontológico e Comportamento de } \\
\text { Retorno de Escolares em Função } \\
\text { do Tipo de Instituição (Pública ou } \\
\text { Privada). 2010. São Paulo. }\end{array}$ & $\begin{array}{l}10-15 \\
\text { Não } \\
\text { informaram } \\
\text { o sexo }\end{array}$ & $\begin{array}{l}\text { Avaliar o conhecimento } \\
\text { odontológico e } \\
\text { comportamento de retorno de } \\
\text { escolares em função do tipo } \\
\text { de instituição (pública ou } \\
\text { privada }\end{array}$ & $\begin{array}{c}\text { Os adolescentes apresentaram conhecimento } \\
\text { odontológico e comportamento de retorno } \\
\text { distintos, segundo o tipo de instituição de } \\
\text { ensino }\end{array}$ & 5 \\
\hline 14 & $\begin{array}{l}\text { Granville-Garcia et al., } \\
\text { Adolescents' knowledge of oral } \\
\text { health: a population-based study, } \\
\text { 2010.Paraíba. }\end{array}$ & $\begin{array}{c}10-19 \\
\text { Mas:311 } \\
\text { Fem: } 368\end{array}$ & $\begin{array}{l}\text { Avaliar o conhecimento em } \\
\text { saúde bucal de adolescentes } \\
\text { de } 10 \text { a } 19 \text { anos da cidade de } \\
\text { Campina Grande, PB, região } \\
\text { Nordeste do Brasil. }\end{array}$ & $\begin{array}{c}\text { Os adolescentes conheciam doenças dentárias } \\
(81 \%) \text {, escovavam os dentes } 3 \text { a } 4 \text { vezes ao dia } \\
(50 \%) ; 51 \% \text { afirmaram usar o fio dental } 57 \% \\
\text { dos adolescentes consultou o dentista no } \\
\text { último ano, sendo a prevenção o motivo mais } \\
\text { frequente }(50 \%) \text {. }\end{array}$ & 5 \\
\hline 15 & $\begin{array}{c}\text { Jordão, Malta, Freire., } \\
\text { Simultaneidade de comportamentos } \\
\text { de risco à saúde bucal em } \\
\text { adolescentes: evidência da } \\
\text { Pesquisa Nacional de Saúde do } \\
\text { Escolar, 2018. Goiás. }\end{array}$ & $\begin{array}{c}10-16 \\
\text { Mas: } 52.012 \\
\text { Fem: } 57.089\end{array}$ & $\begin{array}{l}\text { Identificar a prevalência e a } \\
\text { presença simultânea de } \\
\text { comportamentos de risco à } \\
\text { saúde bucal em adolescentes } \\
\text { brasileiros e fatores } \\
\text { associados }\end{array}$ & $\begin{array}{l}\text { A ocorrência de dois ou mais comportamentos } \\
\text { de risco à saúde bucal foi de } 60,40 \% \text {. As } \\
\text { combinações mais prevalentes com razão } \\
\text { PO/PE acima de } 1,20 \text { foram EDF, CFA e } \\
\text { EDFA. A chance de concentrar dois ou mais } \\
\text { comportamentos foi maior para adolescentes } \\
\text { sem supervisão familiar dos deveres de casa, } \\
\text { de escolas públicas, do sexo masculino e das } \\
\text { raças indígena ou amarela O nível de afluência } \\
\text { familiar baixo atuou como risco para a } \\
\text { combinação ECDFA (OR = 2,58; } \mathrm{p}=0,009) \text {, } \\
\text { enquanto para a combinação CFA os níveis } \\
\text { médio e baixo atuaram como proteção }(\mathrm{OR}= \\
0,71 ; \mathrm{p}<0,001 \text { e OR }=0,76 ; \mathrm{p}=0,011) \text {. }\end{array}$ & 5 \\
\hline 16 & $\begin{array}{l}\text { Mail, Donassoll, Donassollo., } \\
\text { Malocclusion Diagnosis: } \\
\text { Normative Criteria and Self- } \\
\text { Perception of Adolescents. } 2015 . \\
\text { Rio Grande do Sul. }\end{array}$ & $\begin{array}{c}12 \\
\text { Não } \\
\text { informaram } \\
\text { o sexo }\end{array}$ & $\begin{array}{l}\text { Estimar a prevalência de má } \\
\text { oclusão, gravidade, } \\
\text { necessidades de tratamento e } \\
\text { autopercepção em um grupo } \\
\text { de estudantes. }\end{array}$ & $\begin{array}{l}\text { A presença de má oclusão prevaleceu sobre a } \\
\text { oclusão normal, e o overjet maxilar foi a má } \\
\text { oclusão mais prevalecente. No entanto, a } \\
\text { percepção dos adolescentes era maior em } \\
\text { comparação com critérios normativos, o que } \\
\text { sugere que pequenos problemas oclusais são } \\
\text { rapidamente percebidos pelos adolescentes }\end{array}$ & 5 \\
\hline 17 & $\begin{array}{c}\text { Marin et al., Percepção e } \\
\text { informação sobre saúde bucal: } \\
\text { Estudo com adolescentes de uma } \\
\text { escola pública. 2015. Santa } \\
\text { Catarina. }\end{array}$ & $\begin{array}{l}14-15 \\
\text { Mas: } 65 \\
\text { Fem: } 79\end{array}$ & $\begin{array}{c}\text { Analisar o conhecimento e a } \\
\text { percepção sobre saúde bucal } \\
\text { de adolescentes matriculados } \\
\text { no último ano do ensino } \\
\text { fundamental de um } \\
\text { município inserido na região } \\
\text { da Grande Florianópolis } \\
\end{array}$ & $\begin{array}{l}\text { 82\% dos adolescentes informaram que a última } \\
\text { consulta odontológica } \\
\text { ocorreu a menos de um ano e o principal } \\
\text { motivo foi rotina/prevenção e } 90 \% \text { receberam } \\
\text { informações sobre saúde bucal }\end{array}$ & 5 \\
\hline
\end{tabular}




\begin{tabular}{|c|c|c|c|c|c|}
\hline & & & (Santa Catarina, Brasil). & & \\
\hline 18 & $\begin{array}{l}\text { Massoni et al., Dor de dentes e } \\
\text { fatores associados entre } \\
\text { adolescentes de um município de } \\
\text { grande porte populacional no } \\
\text { Nordeste brasileiro. 2020. Paraíba. }\end{array}$ & $\begin{array}{l}10-19 \\
\text { Mas: } 123 \\
\text { Fem:335 }\end{array}$ & $\begin{array}{l}\text { Descrever e analisar o } \\
\text { histórico de dor de dente e } \\
\text { seus fatores associados, entre } \\
\text { adolescentes de escolas } \\
\text { públicas de um município do } \\
\text { Nordeste do Brasil. }\end{array}$ & $\begin{array}{c}\text { A prevalência de dor de dente na vida foi alta } \\
\text { entre os adolescentes de menor idade }(76,9 \% ; \mathrm{p} \\
=0,004) \text {, que visitaram o Cirurgião-Dentista } \\
\text { alguma vez }(74,8 \% ; \mathrm{p}=0,001) \text { e que relataram } \\
\text { medo frente ao atendimento odontológico } \\
(74,9 \% ; \mathrm{p}=0,006) \text {. Conclui-se que a } \\
\text { prevalência de dor de dente na vida tem } \\
\text { associação significativa com os fatores: sexo, } \\
\text { idade, visita ao Cirurgião-Dentista e sensação } \\
\text { de medo. }\end{array}$ & 5 \\
\hline 19 & $\begin{array}{l}\text { Mauricio, Moreira., Oral health } \\
\text { status of the ethnic group Xukuru } \\
\text { from Ororubá: multilevel analysis. } \\
\text { 2014. Pernambuco. }\end{array}$ & $\begin{array}{c}\text { 10-14 } \\
\text { Mas: } 121 \\
\text { Fem: } 112\end{array}$ & $\begin{array}{l}\text { Analisar a situação de saúde } \\
\text { bucal dos indígenas Xukuru } \\
\text { do Ororubá de } 10 \text { a } 14 \text { anos, } \\
\text { em Pernambuco, Brasil.. }\end{array}$ & $\begin{array}{c}\text { Verificou-se que a ausência de cárie está } \\
\text { associada a aspectos contextuais e individuais } \\
\text { do povo indígena Xukuru do Ororubá na faixa } \\
\text { etária de } 10 \text { a } 14 \text { anos. }\end{array}$ & 5 \\
\hline 20 & $\begin{array}{l}\text { Pilleco et al., Factors associated } \\
\text { with the number of filled teeth in } \\
\text { adolescents from public schools: a } \\
\text { cohort study. } 2020 \text {. Rio Grande do } \\
\text { Sul. }\end{array}$ & $\begin{array}{c}12 \\
\text { Mas: } 524 \\
\text { Fem: } 610\end{array}$ & $\begin{array}{l}\text { Avaliar a associação das } \\
\text { condições demográficas, } \\
\text { nível socioeconômico, } \\
\text { variáveis clínicas e fatores } \\
\text { psicossociais com o número } \\
\text { de dentes obturados em } \\
\text { adolescentes de escolas } \\
\text { públicas }\end{array}$ & $\begin{array}{l}\text { O número de dentes obturados foi influenciado } \\
\text { por variáveis clínicas e fatores psicossociais. O } \\
\text { tratamento restaurador é uma intervenção } \\
\text { conservadora e eficaz, que pode resolver } \\
\text { problemas bucais em adolescentes }\end{array}$ & 4 \\
\hline 21 & $\begin{array}{c}\text { Silveira et al., Tooth decay and } \\
\text { associated factors among } \\
\text { adolescents in the north of the State } \\
\text { of Minas Gerais, Brazil: a } \\
\text { hierarchical analysis. 2015. Minas } \\
\text { Gerais. }\end{array}$ & $\begin{array}{c}15-19 \\
\text { Mas:367 } \\
\text { Fem:396 }\end{array}$ & $\begin{array}{c}\text { Descrever as condições de } \\
\text { saúde bucal relacionada à } \\
\text { cárie dentária de adolescentes } \\
\text { dessa região, bem como } \\
\text { identificar os seus potenciais } \\
\text { determinantes }\end{array}$ & $\begin{array}{c}\text { As prevalências de cárie dentária, dente } \\
\text { cariado, restaurado e perdido foram } 71,3 \% \text {, } \\
36,5 \%, 55,6 \% \text { e } 16, \% \text {, respectivamente } \\
\text { Os fatores determinantes para carie foram: ser } \\
\text { negro/índio/pardo, de famílias com maior } \\
\text { aglomeração, não ir ao dentista regularmente, } \\
\text { ter usado serviços públicos e filantrópicos, ser } \\
\text { tabagista, usar álcool e não ter percepção de } \\
\text { saúde bucal. }\end{array}$ & 5 \\
\hline 22 & $\begin{array}{l}\text { Vazquez et al., Individual and } \\
\text { contextual factors related to dental } \\
\text { caries in underprivileged Brazilian } \\
\text { adolescents. } 2015 \text { São Paulo. }\end{array}$ & $\begin{array}{c}15-19 \\
\text { Mas: } 520 \\
\text { Fem:659 }\end{array}$ & $\begin{array}{l}\text { Identificar as variáveis } \\
\text { individuais e contextuais } \\
\text { relacionadas à cárie em } \\
\text { adolescentes carentes da } \\
\text { região sudeste do Brasil, e } \\
\text { para estudar a disparidade da } \\
\text { distribuição da doença. }\end{array}$ & $\begin{array}{l}\text { Apresentaram mais caries os que tinham } \\
\text { presidiário na família e moram em domicílios } \\
\text { com muitas pessoas. } \\
\text { O CPOD foi menor nos bairros com maior } \\
\text { acesso a } \\
\text { esgoto doméstico, e o número de dentes } \\
\text { cariados foi maior nos bairros com os piores } \\
\text { índices de exclusão social. }\end{array}$ & 5 \\
\hline 23 & $\begin{array}{l}\text { Veiga et al., Impact of oral health } \\
\text { and body image in school bullying. } \\
\text { 2019. Bahia. }\end{array}$ & $\begin{array}{l}12-15 \\
\text { Não informou o } \\
\text { sexo }\end{array}$ & $\begin{array}{c}\text { Verificar a associação entre } \\
\text { saúde oral, imagem corporal } \\
\text { e experiência de bullying em } \\
\text { uma população de } \\
\text { adolescentes de escolas } \\
\text { públicas de uma localidade } \\
\text { urbana no nordeste do Brasil. }\end{array}$ & $\begin{array}{l}\text { Foi observada uma alta prevalência de } \\
\text { estudantes afetados pelo bullying. Foi possível } \\
\text { observar uma associação entre o bullying e a } \\
\text { insatisfação com a saúde bucal e imagem } \\
\text { corporal. }\end{array}$ & 5 \\
\hline
\end{tabular}

Fonte: Autores.

Os textos foram organizados em uma classificação dos eixos temáticos abordados pelos estudos nos adolescentes brasileiros. 50\% ( $\mathrm{n}=11)$ dos artigos destacavam necessidade de acesso aos serviços de saúde bucal (da Fonseca et al., 2017; Dutra et al., 2019; Dutra, 2020; Ely et al., 2016; Emerich et al., 2015; Fontanini et al., 2015; Pilecco et al., 2020; Silveira et al., 2015; Vazquez Fde et al., 2015; Veiga da Silva Siqueira et al., 2019); 36\% ( $n=8)$ evidenciaram a prevalência e necessidades de cuidados com a saúde bucal (Aguiar et al., 2014; Assaf et al., 2014; Bendo et al., 2012; Cunha \& Pereira, 2019; Jordão et al., 2018; Mail et al., 2015; Massoni et al., 2020; Mauricio Hde \& Moreira Rda, 2014) e 17\% ( $n=4)$ dos estudos selecionados destacaram os conhecimentos e atitudes dos adolescentes em relação as condições de saúde bucal (Dutra et al., 2019; Fonseca et al., 2014; Garcia et al., 2010; Marín et al., 2016).

Ademais, em relação as regiões brasileiras em que os estudos foram desenvolvidos pode-se perceber que, as regiões Nordeste e Sudeste foram responsáveis por 16 artigos (70\%) da publicação em todo o Brasil. Além disso, a região Norte não apresentou nenhuma publicação sobre a temática atuação do cirurgião-dentista no atendimento à adolescentes no Brasil. 


\section{Discussão}

A adolescência é o período em que as condições bucais mais prevalentes ainda dependem da presença de um biofilme patogênico (Souza \& Roncalli, 2007; Dutra, 2020). Essa afirmativa está estritamente relacionada a negligência da higiene bucal e a presença de uma dieta rica em carboidratos adotada nessa faixa etária da população (Aguiar et al., 2014; Vazquez Fde et al., 2015). Dessa forma, serão discutidos os perfis dos adolescentes brasileiros, fatores socioeconômicos, quais são as comorbidades orais mais recorres, atuação do cirurgião dentista na atenção básica e o papel da Odontologia no atendimento à adolescentes no Brasil.

A análise do perfil demográfico do atendimento à adolescentes no Brasil revela que a amostra foi predominantemente de indivíduos do sexo feminino. Esses resultados vão ao encontro de estudos desenvolvidos por Bendo (2012) onde as mulheres apresentaram melhores panoramas sobre as condições de saúde bucal e sistêmica, preocupação com a estética ou pelos padrões exigidos pela sociedade, além de serem em maior número na população (Bendo et al., 2012).

A maioria dos adolescentes que apresentavam maior risco as doenças bucais como, por exemplo, a cárie, a doença periodontal e as mal oclusões foram aqueles indivíduos que se autodeclararam de cor parda ou preta. Como também grande parte deles residiam em domicílios com maior número de pessoas e com questões de vulnerabilidade socioeconômica como demonstrado nos artigos eleitos por esta revisão integrativa de literatura (Aguiar et al., 2014; Cunha \& Pereira, 2019; Dutra et al., 2019; Dutra, 2020; Emerich et al., 2015).

O Índice de Desenvolvimento Humano (IDH) está ligado ao alto risco da experiência de cárie dentária e há concordância entre os estudos quando citam que a condição socioeconômica afeta diretamente na incidência e prevalências de cáries dentárias. Isso se deve pelo fato de no Brasil haver uma grande desigualdade social e que a maior parte da população não tem condições nem de suprir suas necessidades básicas. Com isso, a saúde bucal passa a ser a menor preocupação (Roncalli, 2010Assaf et al., 2014; Emerich et al., 2015; Pilecco et al., 2020; Silveira et al., 2015; Vazquez Fde et al., 2015). Somado a isso, um estudo realizado Fontanini, Marshman, Vettore (2015), investigaram o papel do apoio social e rede social com a cárie dentária em adolescentes utilizando os conceitos dos determinantes sociais de saúde da Organização Mundial de Saúde. Seus resultados identificaram que o número de redes sociais e as condições socioeconômicas influenciaram na saúde bucal dos adolescentes em uma região do Centro-Oeste do Brasil (Fontanini et al., 2015).

Diante das diversas doenças bucais identificadas pelos estudos incluídos a cárie dentária foi a mais prevalente. Considerada uma doença infectocontagiosa de lenta progressão e de causa multifatorial (depende do hospedeiro, da dieta e da microbiota) para que se estabeleça. E, por esses fatores também é denominada como uma "doença social", fato esse que leva a afirmar que a cárie é um problema de saúde pública que afetam crianças, adolescentes, adultos e idosos em todo mundo (Roncalli, 2010; Assaf et al., 2014; Cunha \& Pereira, 2019; Dutra, 2020).

Ademais, as mal oclusões também foram identificadas por esta revisão integrativa como uma condição que influencia negativamente na qualidade de vida destes adolescentes (Ely et al., 2016; Mail et al., 2015; Marín et al., 2016; Mauricio Hde \& Moreira Rda, 2014; Pilecco et al., 2020). De acordo com Mail, Donassoll, Donassollo (2015) a presença da mal oclusão foram predominantemente identificada nos adolescentes do Rio Grande do Sul, como também foi considerado um problema que é rapidamente percebido por eles (Mail, Donassoll, Donassol, 2015). Esses resultados puderam ser endossados por Veiga (2019) em que avaliaram o impacto da saúde bucal e da imagem corporal no bullying escolar e, seus resultados demonstraram uma significativa associação entre o bullying e a prevalência de cárie dentária, doença periodontal e a mal oclusão nos adolescentes da Bahia (Veiga et al., 2019).

Neste contexto, pesquisas validam a importância do cirurgião-dentista no trabalho interdisciplinar, acima de tudo, a intersetorialidade em todos os âmbitos tanto em escolas como na atenção básica de saúde. Outro ponto importante evidenciado por nosso estudo foi a importância da educação em saúde no ambiente escolar, uma vez que, vários estudos associam o grau de 
instrução/escolaridade dos familiares com a experiência de cáries em adolescentes e crianças (Correa et al., 2012; Ely et al., 2016). Posto isso, os artigos que compuseram a amostra final desta revisão destacaram a relação entre o estabelecimento de vínculo dos adolescentes com acesso aos serviços públicos de saúde odontológico como fator crucial para a adesão do tratamento. Visto que, tais ações facilitarão as orientações realizadas sobre os cuidados com a saúde bucal, gerando assim, maior efetividade nas ações de promoção à saúde tanto no âmbito da estratégia da saúde da família (ESF) quanto nas escolas (Cunha \& Pereira, 2019; da Fonseca et al., 2017; Dutra et al., 2019; Granville-Garcia et al., 2010).

Estudos afirmam que os adolescentes não procuram o atendimento odontológico (atenção primária de saúde ou do setor privado de saúde) por não apresentarem uma autopercepção aguçada sobre suas condições de saúde bucal, do mesmo modo que relatam que a participação dos adolescentes em atividades educativas e de prevenção em saúde bucal apresentaram-se reduzidas quando comparadas com outras faixas etárias (Assaf et al., 2014; Mauricio Hde \& Moreira Rda, 2014; Silveira et al., 2015). No entanto, Silveira e colaboradores (2015) demostram que os adolescentes que utilizam os serviços públicos de saúde apresentam maiores índices de cárie dentária quando comparado com aqueles que utilizam os serviços privados/plano de saúde (Silveira et al., 2015).

Grande parte dos estudos evidenciados nesta revisão integrativa sugerem a necessidade de acesso aos serviços odontológicos dos adolescentes no Brasil. Isso pode ser observado no estudo desenvolvido por Silveira (2015), onde as famílias de vulnerabilidade social não procuram regulamente ao dentista para o tratamento preventivo da cárie ou da doença periodontal (Silveira et al., 2015). Ademais, os artigos incluídos evidenciaram a necessidade de associar a prática curativista com a preventiva no atendimento odontológico visto que, o desenvolvimento da autonomia de seus pacientes acerca do processo saúde-doença torna-se fundamental na qualidade de vida dos mesmos e o cirurgião-dentista desenvolve ações de promoção e prevenção de saúde bucal no atendimento à adolescentes no Brasil.

Por fim, destaca-se como limitação parcial deste estudo a inclusão da literatura cinzenta, teses e dissertações. Falamos em limitações parciais, pois, os artigos incluídos nessa revisão integrativa de literatura permitiram delinear como o papel da Odontologia no atendimento à adolescentes no Brasil.

\section{Conclusão}

Verificou-se que mesmo com a evolução dos padrões de saneamento do país as condições bucais dos adolescentes ainda requerem cuidados e que o atendimento está relacionado a queixa do adolescente, ou seja, a dor de dente, a carie e infecções gengivais. Diante disso, é necessário que os centros de pesquisas multicêntricas e estratégia de saúde da família e escolas adotem medidas preventivas e de promoção em saúde considerando os indicadores socioeconômicos de cada região promovendo assim, o elo entre o cirurgião-dentista e o paciente adolescente.

Os municípios devem estar atentos ao implantarem os serviços de saúde bucal de realizar atividades para os adolescentes, assim como as instituições de ensino que utilizam os PSF para práticas e estágios, pois a saúde bucal preveni várias doenças sistêmicas. Acredita-se que esse estudo contribui para alertar a importância da saúde bucal para os adolescentes e abre uma demanda para verificar porquê as mulheres chegam com menos cáries do que os homens.

\section{Contribuições}

Todos os autores participaram substancialmente na concepção, planejamento do estudo, redação e revisão crítica. E, todos os autores aprovaram a versão a ser publicada e assumem responsabilidade por todos os aspectos do trabalho. 


\section{Referências}

Aguiar, Y. P., dos Santos, F. G., Moura, E. F., da Costa, F. C., Auad, S. M., de Paiva, S. M., \& Cavalcanti, A. L. (2014). Association between dental erosion and diet in Brazilian adolescents aged from 15 to 19: a population-based study. Scient World Journal, 2014, 818167. https://doi.org/10.1155/2014/818167

Assaf, A. V., Caldo-Teixeira, A. S., Silveira, F. M., Valente, M. I. B., Ditterich, R. G., \& Barcelos, R. (2014). Dental caries in inland Brazilian adolescents and its relationship with social determinants. Braz. j. oral sci, 13, 133-139.

Bendo, C. B., Vale, M. P., Figueiredo, L. D., Pordeus, I. A., \& Paiva, S. M. (2012). Social vulnerability and traumatic dental injury among Brazilian schoolchildren: a population-based study. Int J Environ Res Public Health, 9, 4278-4291. https://doi.org/10.3390/ijerph9124278

Correa, C. R. S., Saintrain, M. V. d. L., \& Vieira, A. P. G. F. (2012). Saúde bucal do adolescente: necessidades odontológicas levantadas pelos agentes comunitários de saúde. Rev. bras. promoç. saúde (Impr.), 25.

Cunha, I. P. D., \& Pereira, A. C. (2019). Association between social conditions and oral health in school failure. 53, 108. https://doi.org/10.11606/s15188787.2019053001457

da Fonseca, E. P., Frias, A. C., Mialhe, F. L., Pereira, A. C., \& Meneghim, M. D. (2017). Factors associated with last dental visit or not to visit the dentist by Brazilian adolescents: A population-based study. PLoS One, 12, Article e0183310. https://doi.org/10.1371/journal.pone.0183310

Dutra, L. D., de Lima, L. C. M., Neves, E. T. B., Gomes, M. C., de Araujo, L. J. S., Forte, F. D. S., \& Granville-Garcia, A. F. (2019). Adolescents with worse levels of oral health literacy have more cavitated carious lesions. PLoS One, 14, Article e0225176. https://doi.org/10.1371/journal.pone.0225176

Dutra, L. D. C. (2020). Degree of family cohesion and social class are associated with the number of cavitated dental caries in adolescents. Braz Oral Res, 34, e037. https://doi.org/10.1590/1807-3107bor-2019.vol33.012410.1590/1807-3107bor-2020.vol34.0037

Ely, H. C., Abegg, C., Celeste, R. K., \& Pattussi, M. P. (2016). Impacto das equipes de saúde bucal da Estratégia da Saúde da Família na saúde bucal de adolescentes do sul do Brasil. Cien Saude Colet, 21, 1607-1616. https://doi.org/10.1590/1413-81232015215.07822015

Emerich, T. B., Pacheco, K. T. d. S., Carvalho, R. B. d., Muniz, E. N., Sarcinelli, G. P., \& Sarti, T. D. (2015). Access to Dental Services and Related Factors in Adolescents from Vitória, Espírito Santo, Brazil, 2011. Pesqui. bras. odontopediatria clín. integr, 15, 253-262. http://revista.uepb.edu.br/index.php/pboci/article/view/2910/pdf

Federal, G. J. L. f. (1990). Estatuto da Criança e do Adolescente. 8.

Fonseca, B. B., Wapniarz, R. S., \& Torres-Pereira, C. C. (2014). Atitudes e acesso à informação de saúde bucal de um grupo de gestantes adolescentes. Rev. Assoc. Paul. Cir. Dent, 68, 254-258. https://pesquisa.bvsalud.org/portal/resource/pt/lil-729360

Fontanini, H., Marshman, Z., \& Vettore, M. (2015). Social support and social network as intermediary social determinants of dental caries in adolescents. Community Dent Oral Epidemiol, 43, 172-182. https://dx.doi.org/10.1111/cdoe.12139

Garcia, P. P. N. S., Campos, J. A. D. B., Cezare, L., Bonan, R. F., Pinto, L. d. R., \& Derceli, J. d. R. (2010). Conhecimento odontologico e comportamento de retorno de escolares em funcao do tipo de instituicao (pública ou privada). Pesqui. bras. odontopediatria clín. integr, 10 . http://revista.uepb.edu.br/index.php/pboci/article/view/939/445

Granville-Garcia, A. F., Fernandes, L. V., Farias, T. S. S. d., Avila, S., Cavalcanti, A. L., \& Menezes, V. A. (2010). Conhecimento do adolescente em relação a saúde bucal: um estudo de base populacional. Rev. odonto ciênc, 25.

Jordão, L. M. R., Malta, D. C., \& Freire, M. d. C. M. (2018). Simultaneidade de comportamentos de risco à saúde bucal em adolescentes: evidência da Pesquisa Nacional de Saúde do Escolar. 21, e180019.

Roncalli, Angelo Giuseppe. "Projeto SB Brasil 2010-pesquisa nacional de saúde bucal revela importante redução da cárie dentária no país." (2011): 4-5.

Jordão, L. M. R., Malta, D. C., \& Freire, M. D. C. M. (2018). Simultaneous oral health risk behaviors among adolescents: evidence from the National Schoolbased Student Health Survey.]. Rev Bras Epidemiol, 21(suppl 1), e180019-e180019.

Mail, L. R., Donassollo, S. H., \& Donassollo, T. A. (2015). Malocclusion Diagnosis: Normative Criteria and Self-Perception of Adolescents. Pesquisa Bras. Em Odontoped. E Clin. Integ., 15(1), 197-203. https://doi.org/10.4034/pboci.2015.151.21

Marín, C., Papadopol, P. M., Bottan, E. R., \& Orcina, B. F. (2016). Percepção e informação sobre saúde bucal: estudo com adolescentes de uma escola pública. Saude e pesqui. (Impr.), 9, 499-506.

Massoni, A. C. d. L. T., Porto, É., Ferreira, L. R. B. O., Gomes, M. d. N. C., Granville-Garcia, A. F., \& Avila, S. (2020). Dor de dentes e fatores associados entre adolescentes de um município de grande porte populacional no Nordeste brasileiro. Ciênc. Saúde Colet, 25, 673-682.

Mauricio Hde, A., \& Moreira Rda, S. (2014). Oral health status of the ethnic group Xukuru from Ororubá: multilevel analysis. Rev Bras Epidemiol, 17, 787800. https://doi.org/10.1590/1809-4503201400030017

Melnyk, B. M., \& Fineout-Overholt, E. (2011). Evidence-based practice in nursing \& healthcare: A guide to best practice. Lippincott Williams \& Wilkins.

Mendes, K. D. S., Silveira, R. C. d. C. P., \& Galvão, C. M. (2008). Revisão integrativa: método de pesquisa para a incorporação de evidências na saúde e na enfermagem. Texto \& contexto enfermagem, 17, 758-764.

Organization, W. H. Adolescent health. URL: http://www.who.int/topics/adolescent_health/en/

Pilecco, R. O., Godois, L. d. S., Maroneze, M. C., Ortiz, F. R., \& Ardenghi, T. M. (2020). Factors associated with the number of filled teeth in adolescents from public schools: a cohort study. Braz Oral Res, 33, e124-e124. 
Research, Society and Development, v. 10, n. 15, e188101522244, 2021

(CC BY 4.0) | ISSN 2525-3409 | DOI: http://dx.doi.org/10.33448/rsd-v10i15.22244

Santos C.M.C., Pimenta C.A.M, \& Nobre M.R.C. (2007). A Estratégia PICO Para A Construção Da Pergunta De Pesquisa e busca de Evidências. Rev Latinoam Enfermagem 15.

Spezzia s., Lamelo, K.M.S.M.; Jahn, R.S.; \& Jahn M.R. O papel da Odontopediatria na saúde bucal do adolescente. Conselho Paulista de Odontologia.

Silveira, M. F., Freire, R. S., Nepomuceno, M. O., Martins, A., \& Marcopito, L. F. (2015). Tooth decay and associated factors among adolescents in the north of the State of Minas Gerais, Brazil: a hierarchical analysis. Cien Saude Colet, 20, 3351-3364. https://doi.org/10.1590/1413-812320152011.12262014

Souza, T. M. S. d., \& Roncalli, A. G. (2007). Saúde bucal no Programa Saúde da Família: uma avaliação do modelo assistencial. Cad Saude Publica, 23, 27272739 .

Taquette, S. R. (2010). Conduta ética no atendimento à saúde de adolescentes. Adolescencia e Saude, 7, 6-11.

Ursi, E. S. (2005). Prevenção de lesões de pele no perioperatório: revisão integrativa da literatura [dissertação]. Ribeirão Preto: Universidade de São Paulo, Escola de Enfermagem de Ribeirão Preto.

Vazquez Fde, L., Cortellazzi, K. L., Kaieda, A. K., Bulgareli, J. V., Mialhe, F. L., Ambrosano, G. M., \& Pereira, A. C. (2015). Individual and contextual factors related to dental caries in underprivileged Brazilian adolescents. BMC Oral Health, 15, 6. https://doi.org/10.1186/1472-6831-15-6

Veiga da Silva Siqueira, D., Dos Santos, I. M., Pereira, L. L., Leal Tosta Dos Santos, S. C., Cristino, P. S., Pena Messias de Figueiredo Filho, C. E., \& Figueiredo, A. L. (2019). Impact of oral health and body image in school bullying. Spec Care Dentist, 39, 375-379. https://doi.org/10.1111/scd.12401 